\title{
Continuity versus boundedness of the spectral factorization mapping
}

\author{
by \\ Holger Boche (Berlin) and Volker Pohl (Haifa)
}

\begin{abstract}
This paper characterizes the Banach algebras of continuous functions on which the spectral factorization mapping $\mathfrak{S}$ is continuous or bounded. It is shown that $\mathfrak{S}$ is continuous if and only if the Riesz projection is bounded on the algebra, and that $\mathfrak{S}$ is bounded only if the algebra is isomorphic to the algebra of continuous functions. Consequently, $\mathfrak{S}$ can never be both continuous and bounded, on any algebra under consideration.
\end{abstract}

1. Introduction. The operation by which a function $f$ on the unit circle is written as $f(\zeta)=f_{+}(\zeta) \overline{f_{+}(\zeta)}$ for all $\zeta \in \mathbb{T}:=\{z \in \mathbb{C}:|z|=1\}$, with an outer function $f_{+}$, is known as spectral factorization. This operation arises in many different applications, e.g. in communications [16], estimation [10], or control theory [6], but it is also of theoretical use in the area of stochastic processes $[4,13]$ and in the theory of singular integral equations $[3,17]$. A survey of methods for calculating the spectral factor $f_{+}$can be found in [15].

The continuity and boundedness of the spectral factorization mapping $\mathfrak{S}: f \mapsto f_{+}$is of vital interest in many applications in order to control errors which arise due to disturbances in the spectral data. Since $\mathfrak{S}$ is a non-linear operator, its boundedness does not imply its continuity and vice versa, and the question whether $\mathfrak{S}$ is bounded or continuous depends crucially on the normed space one is using. Recently, there was some interest in the relation between the boundedness and continuity of other non-linear operators on various spaces. For example, [11] characterized spaces on which the HardyLittlewood maximal operator is bounded, whereas in [12] the continuity of the maximal operator was investigated on Sobolev spaces. These works

2000 Mathematics Subject Classification: Primary 47A68, 47H99, 46J10; Secondary 46J15.

Key words and phrases: spectral factorization, boundedness, continuity, non-linear operators.

The work of the first author was partly supported by the German Research Foundation (DFG) under Grant BO 1734/11-2.

The second author was supported by DFG under Grant PO 1347/1-1. 
show that there exist spaces on which the maximal operator is bounded and continuous, whereas on other spaces it has only one of these properties.

The spectral factorization mapping shows yet a different behavior. A major difficulty is that its domain is unknown in general, and that there may not exist a closed form representation of $\mathfrak{S}$ for all elements in its domain. However, to investigate the boundedness of $\mathfrak{S}$, the present paper first considers $\mathfrak{S}$ on the set of all polynomials, for which the spectral factorization is given by a theorem of Fejér-Riesz. For the investigation of the continuity of $\mathfrak{S}$, we first assume only that the spectral factorization exists in a neighborhood of the unity of the algebra. Based on the considerations on these subsets, it is shown that the continuity of the spectral factorization mapping implies its unboundedness, and conversely its boundedness implies its discontinuity on all Banach algebras under consideration. These are algebras of continuous functions on the unit circle, which are defined by four axioms $(B 1)-(B 4)$ in Section 3 and which we name $\mathcal{S}$-algebras. These axioms are similar to those introduced by Peller and Khrushchev [13] in the context of best approximation of analytic functions. Algebras satisfying the axioms of Peller and Khrushchev are called decomposing Banach algebras [3, 9], and Jacob and Partington [9] studied the spectral factorization mapping on such algebras in detail. The class of $\mathcal{S}$-algebras, considered in the present paper, contains the class of decomposing Banach algebras, but $\mathcal{S}$-algebras need not be decomposing, in the sense that the Riesz projection is not necessarily bounded on these algebras. It was proved in [9] and [2] respectively that $\mathfrak{S}$ is continuous and unbounded on every decomposing Banach algebra. In the present paper, we will see that the decomposing algebras are precisely those $\mathcal{S}$-algebras on which $\mathfrak{S}$ is continuous. On the other hand, if $\mathfrak{S}$ is bounded on an $\mathcal{S}$-algebra then the algebra has to be isomorphic to the algebra $\mathcal{C}(\mathbb{T})$ of continuous functions.

2. Spectral factorization. Throughout this paper, $\mathbb{D}:=\{z \in \mathbb{C}$ : $|z|<1\}$ and $\mathbb{T}:=\{z \in \mathbb{C}:|z|=1\}$ stand for the unit disc and unit circle in the complex plane, respectively. The imaginary unit is denoted by $i=\sqrt{-1}$ and $\bar{z}$ is the complex conjugate of $z$. The $\operatorname{set} \mathcal{C}(\mathbb{T})$ of all continuous functions on $\mathbb{T}$ is a Banach space under the usual supremum norm $\|f\|_{\infty}=$ $\sup _{\zeta \in \mathbb{T}}|f(\zeta)|$, and $\mathcal{P}(N)$ is the set of all trigonometric polynomials of degree of at most $N$. The Banach space of $p$-integrable functions $(1 \leq p<\infty)$ on $\mathbb{T}$ with the usual norm $\|f\|_{p}:=\left((2 \pi)^{-1} \int_{\mathbb{T}}|f(\zeta)|^{p} d \zeta\right)^{1 / p}$ is denoted by $L^{p}(\mathbb{T})$, and $L^{\infty}(\mathbb{T})$ denotes the set of all measurable and essentially bounded functions on $\mathbb{T}$. For every $f \in L^{1}(\mathbb{T})$, the Fourier coefficients are defined by

$$
\widehat{f}(k)=\frac{1}{2 \pi} \int_{-\pi}^{\pi} f\left(e^{i \tau}\right) e^{-i k \tau} d \tau \quad(k=0, \pm 1, \pm 2, \ldots) .
$$


For a commutative Banach algebra $\mathcal{A} \subset L^{1}(\mathbb{T})$, we define

$$
\mathcal{A}_{+}:=\{f \in \mathcal{A}: \widehat{f}(k)=0 \text { for all } k<0\} .
$$

In particular $\left[L^{p}(\mathbb{T})\right]_{+}, 0 \leq 1 \leq \infty$, coincides with the Hardy space $H^{p}(\mathbb{D})$ of analytic functions in $\mathbb{D}$, and every $f \in \mathcal{A}_{+}$can be identified with a function

$$
f(z)=\sum_{k=0}^{\infty} \widehat{f}(k) z^{k} \quad(z \in \mathbb{D}),
$$

which is holomorphic for all $z \in \mathbb{D}$. For an arbitrary set $\mathcal{A}$ of functions on the unit circle, $\mathcal{A}_{\text {pos }}=\{f \in \mathcal{A}: f(\zeta) \geq 0$ for all $\zeta \in \mathbb{T}\}$ will denote the set of all non-negative functions in $\mathcal{A}$.

There exists a natural projection from $L^{2}(\mathbb{T})$ to $H^{2}(\mathbb{D})$, which will be denoted by

$$
\mathfrak{P}_{+}: \sum_{k=-\infty}^{\infty} \widehat{f}(k) \zeta^{k} \mapsto \sum_{k=0}^{\infty} \widehat{f}(k) \zeta^{k} \quad(\zeta \in \mathbb{T})
$$

and which is called the Riesz projection. Inserting the Fourier coefficients (2.1) into (2.3) yields the integral representation

$$
\left(\mathfrak{P}_{+} f\right)(z)=\frac{1}{2 \pi} \int_{-\pi}^{\pi} f\left(e^{i \tau}\right) \frac{e^{i \tau}}{e^{i \tau}-z} d \tau \quad(z \in \mathbb{D}) .
$$

Let $\mathcal{A} \subset L^{1}(\mathbb{T})$ be a commutative Banach algebra. A linear functional $h: \mathcal{A} \rightarrow \mathbb{C}$ is called a homomorphism (a multiplicative linear functional) on $\mathcal{A}$ if $h(f g)=h(f) h(g)$ for all $f, g \in \mathcal{A}$. The set of all homomorphisms on $\mathcal{A}$ will be denoted by $\Gamma(\mathcal{A})$, and $\mathcal{G}(\mathcal{A})$ stands for the set of all invertible elements of $\mathcal{A}$. Thus $\mathcal{G}(\mathcal{A})$ is the set of all $f \in \mathcal{A}$ for which there exists a function $f^{-1} \in \mathcal{A}$ such that $f f^{-1}=1$, where 1 denotes the unity of $\mathcal{A}$.

For every $f \in \mathcal{A}$ one defines $(\exp f)(\zeta):=\sum_{k=0}^{\infty}(1 / k !) f(\zeta)^{k}$. Since $\mathcal{A}$ is a Banach algebra, it is clear that $\|\exp f\|_{\mathcal{A}} \leq \exp \left(\|f\|_{\mathcal{A}}\right)$, and therefore $\exp f \in \mathcal{A}$, and the common properties of the exponential series imply that $\exp f \in \mathcal{G}(\mathcal{A})$. Moreover, $\exp (\mathcal{A}):=\left\{f=e^{g}: g \in \mathcal{A}\right\}$ is the set all functions $f \in \mathcal{A}$ for which $\log f \in \mathcal{A}$. The logarithm may be defined by the power series $\log (\mathbf{l}-f)=-\sum_{k=1}^{\infty}(1 / k) f^{k}$ for all $f \in \mathcal{A}$ with $\|f\|_{\mathcal{A}}<1$. Based on this power series, one easily verifies

Lemma 2.1. Let $\mathcal{A}$ be commutative Banach algebra with unity, let $f \in$ $\exp (\mathcal{A})$ and $h \in \mathcal{A}$ with $\|h\|_{\mathcal{A}}<\frac{1}{2}\left\|f^{-1}\right\|_{\mathcal{A}}^{-1}$. Then $\log (f-h) \in \exp (\mathcal{A})$ and

$$
\|\log (f-h)-\log (f)\|_{\mathcal{A}} \leq 2\left\|f^{-1}\right\|_{\mathcal{A}}\|h\|_{\mathcal{A}} .
$$

This lemma implies in particular that $\exp (\mathcal{A})$ is an open set.

Definition 2.2. Let $\mathcal{A} \subset L^{1}(\mathbb{T})$ be a Banach algebra of functions on $\mathbb{T}$. An element $f \in \mathcal{A}$ possesses a spectral factorization in $\mathcal{A}$ if there exists an 
outer function $f_{+} \in \mathcal{A}_{+}$such that

$$
f(\zeta)=f_{+}(\zeta) \overline{f_{+}(\zeta)} \quad \text { for all } \zeta \in \mathbb{T} .
$$

The function $f_{+}$is called a spectral factor of $f$ and every $f \in \mathcal{A}$ which possesses a spectral factorization is called a spectral density. The mapping $\mathfrak{S}: f \mapsto f_{+}$is the spectral factorization mapping. The spectral factor $f_{+}$is unique up to a unitary constant.

REMARK 2.3. Note that the above definition, in contrast e.g. to those in $[2,3,9]$, does not require that the spectral factor $f_{+}$is invertible in $\mathcal{A}_{+}$.

The sets $\mathcal{S}(\mathcal{A})$ and $\mathcal{F}(\mathcal{A}):=\{g=\mathfrak{S} f: f \in \mathcal{S}(\mathcal{A})\}$ consist of all spectral densities and all spectral factors in a Banach algebra $\mathcal{A}$, respectively.

Definition 2.4. A Banach algebra $\mathcal{A} \subset L^{1}(\mathbb{T})$ on which the Riesz projection $\mathfrak{P}_{+}: \mathcal{A} \rightarrow \mathcal{A}_{+}$is bounded is said to be decomposing.

Remark 2.5. In $[9,2], \mathcal{S}$-algebras (see Def. 3.1 below) on which the Riesz projection is bounded were called "decomposing Banach algebras". However in the present paper "decomposing" means only that the Riesz projection $\mathfrak{P}_{+}$is bounded, as in [3].

Proposition 2.6. Let $\mathcal{A} \subset L^{1}(\mathbb{T})$ be a Banach algebra which is decomposing. Then every real-valued function $f \in \exp (\mathcal{A})$ possesses a spectral factorization in $\mathcal{A}$, and one spectral factor is given by

$$
\begin{aligned}
f_{+}(z) & =(\mathfrak{S} f)(z) \\
& =\exp \left(\frac{1}{4 \pi} \int_{-\pi}^{\pi} \log f\left(e^{i \tau}\right) \frac{e^{i \tau}+z}{e^{i \tau}-z} d \tau\right) \quad(z \in \mathbb{D}) .
\end{aligned}
$$

Proof. Assume $f \in \exp (\mathcal{A})$ is an arbitrary real-valued function. Then there exists a $g \in \mathcal{A}$ such that $f=\exp (g)$ and the spectral factorization mapping (2.4) becomes

$$
f_{+}(z)=\sqrt{\exp \left(\frac{1}{2 \pi} \int_{-\pi}^{\pi} g\left(e^{i \tau}\right) \frac{e^{i \tau}+z}{e^{i \tau}-z} d \tau\right)} \quad(z \in \mathbb{D}) .
$$

Since $f$ is integrable, the term under the square root is an outer function $[8$, Chapter 5], which shows that $f_{+}$is analytic on $\mathbb{D}$ and $\left|f_{+}(\zeta)\right|^{2}=(\exp g)(\zeta)=$ $f(\zeta)$ for all $\zeta \in \mathbb{T}$. The spectral factorization mapping (2.4) can also be written as

$$
f_{+}(z)=\exp \left[-\frac{1}{2} \widehat{g}(0)\right] \exp \left[\left(\mathfrak{P}_{+} g\right)(z)\right] .
$$

Hence $\|\mathfrak{S} f\|_{\mathcal{A}} \leq \exp \left(-\frac{1}{2}\|g\|_{1}\right) \exp \left\|\mathfrak{P}_{+} g\right\|_{\mathcal{A}}<\infty$ since $\mathfrak{P}_{+}$is assumed to be bounded and $\|\exp g\|_{\mathcal{A}} \leq \exp \|g\|_{\mathcal{A}}$. Consequently, $\mathfrak{S} f \in \mathcal{A}$ for every $f \in \exp (\mathcal{A})$. 
A second class of functions for which the solution of the spectral factorization problem is known is the set of all non-negative trigonometric polynomials on $\mathbb{T}$. In this case, the spectral factorization is equivalent to the following classical result of L. Fejér and M. Riesz:

TheOREM 2.7 (Fejér-Riesz [5]). For every trigonometric polynomial $f \in$ $\mathcal{P}(N)$ with $f(\zeta) \geq 0$ for all $\zeta \in \mathbb{T}$ there exists an analytic polynomial $f_{+} \in$ $\mathcal{P}_{+}(N)$ with $\left|f_{+}(z)\right|>0$ for all $z \in \mathbb{D}$ such that

$$
f(\zeta)=\left|f_{+}(\zeta)\right|^{2}=f_{+}(\zeta) \overline{f_{+}(\zeta)} \quad \text { for all } \zeta \in \mathbb{T} .
$$

This $f_{+} \in \mathcal{P}_{+}(N)$ is uniquely determined by $f$ up to a unitary factor.

It should be noted that also in the case of trigonometric polynomials, the spectral factor can be represented in the form (2.4), since every nonnegative trigonometric polynomial $f$ satisfies the so called Szegö condition $\int_{-\pi}^{\pi} \log f\left(e^{i \tau}\right) d \tau>-\infty$, so that (2.4) is well defined and has the desired properties for every $f \in \mathcal{P}_{\text {pos }}(N)$.

3. Banach algebras of continuous functions. We consider the spectral factorization mapping on Banach algebras $\mathcal{B}$ of continuous functions which are defined by the following four axioms.

Definition 3.1. A commutative Banach algebra $\mathcal{B} \subset L^{1}(\mathbb{T})$ is called an $\mathcal{S}$-algebra if

$(B 1) \mathcal{B}$ is a Banach algebra with respect to pointwise multiplication.

(B2) If $f \in \mathcal{B}$, then $\bar{f} \in \mathcal{B}$.

(B3) The set of all trigonometric polynomials is dense in $\mathcal{B}$.

(B4) Every multiplicative functional on $\mathcal{B}$ is of the form $f \mapsto f(\zeta)$ for some $\zeta \in \mathbb{T}$.

In the following, the symbol $\mathcal{B}$ will always stand for an $\mathcal{S}$-algebra. The axioms $(B 1)-(B 4)$ of an $\mathcal{S}$-algebra imply certain properties of its elements. Some of these properties, which are needed later, are given in the following propositions.

Proposition 3.2. Let $\mathcal{B}$ be an $\mathcal{S}$-algebra. Then every $f \in \mathcal{B}$ with $f(\zeta) \neq 0$ for all $\zeta \in \mathbb{T}$ belongs to $\mathcal{G}(\mathcal{B})$.

Proof. Let $\gamma \in \Gamma(\mathcal{B})$ be an arbitrary multiplicative functional on $\mathcal{B}$. By axiom $(B 4)$ there exists a $\zeta \in \mathbb{T}$ such that $\gamma(f)=f(\zeta)$ for all $f \in \mathcal{B}$. Therefore, $f(\zeta) \neq 0$ for all $\zeta \in \mathbb{T}$ implies $\gamma(f) \neq 0$ for all $\gamma \in \Gamma(\mathcal{B})$, and by the Beurling-Gelfand theorem, $f \in \mathcal{B}$ is invertible in $\mathcal{B}$ if and only if $\gamma(f) \neq 0$ for all $\gamma \in \Gamma(\mathcal{B})[14, \S 11.5]$.

Proposition 3.3. Every $\mathcal{S}$-algebra $\mathcal{B}$ is continuously embedded in $\mathcal{C}(\mathbb{T})$ with

$$
\|f\|_{\infty} \leq\|f\|_{\mathcal{B}} \quad \text { for all } f \in \mathcal{B} \text {. }
$$


Proof. By $(B 1)$ and $(B 4)$, for every $\zeta \in \mathbb{T}$ there exists a $\gamma \in \Gamma(\mathcal{B})$ such that $\gamma(f)=f(\zeta)$ for every $f \in \mathcal{B}$. Since $\|\gamma\| \leq 1$ for each $\gamma \in \Gamma(\mathcal{B})$ (see e.g. $[14, \S 10.7])$, one has $|f(\zeta)|=|\gamma(f)| \leq\|f\|_{\mathcal{B}}$, which implies $\|f\|_{\infty} \leq\|f\|_{\mathcal{B}}$. By (B3), for every $\varepsilon>0$ there exists a trigonometric polynomial $p$ such that $\|f-p\|_{\infty} \leq\|f-p\|_{\mathcal{B}}<\varepsilon$, which proves that $f \in \mathcal{C}(\mathbb{T})$.

Proposition 3.4. Every $\mathcal{S}$-algebra $\mathcal{B}$ is semisimple and there exists a constant $c_{1}>0$ such that

$$
\|\bar{f}\|_{\mathcal{B}} \leq c_{1}\|f\|_{\mathcal{B}} \quad \text { for all } f \in \mathcal{B} .
$$

Proof. The operation $f \mapsto \bar{f}$ is an involution on $\mathcal{B}$. By axiom (B4), every multiplicative functional $\gamma_{\zeta} \in \Gamma(\mathcal{B})$ is defined by a point $\zeta \in \mathbb{T}$ on the unit circle, according to $\gamma_{\zeta}(f):=f(\zeta)$. The null space of such a homomorphism is $\operatorname{ker} \gamma_{\zeta}=\{f \in \mathcal{B}: f(\zeta)=0\}$, and the intersection of all null spaces contains only the zero function:

$$
\bigcap_{\gamma_{\zeta} \in \Gamma(\mathcal{B})} \operatorname{ker} \gamma_{\zeta}=\bigcap_{\zeta \in \mathbb{T}}\{f \in \mathcal{B}: f(\zeta)=0\}=\{f \equiv 0\} .
$$

This shows that $\mathcal{B}$ is a semisimple commutative algebra on which the involution $f \mapsto \bar{f}$ is always continuous $([14, \S 11.16])$.

4. Continuity of the spectral factorization mapping. This section characterizes the $\mathcal{S}$-algebras on which the spectral factorization mapping $\mathfrak{S}$ is continuous. Since the trigonometric polynomials are dense in every $\mathcal{S}$-algebra $\mathcal{B}$, we know from the theorem of Fejér-Riesz that the spectral factorization exists at least for all non-negative trigonometric polynomials in $\mathcal{B}$ and that one spectral factor is given by (2.4). However, since we do not know whether $\mathfrak{S}$ is continuous, it is not clear at the outset whether the spectral factorization exists in $\mathcal{B}$ for non-polynomials. For this reason, we will assume that the spectral factorization exists at least in a neighborhood of the unity $\perp$ of $\mathcal{B}$. Then it is shown that if the spectral factorization mapping is continuous at $\mathbf{1}$, it will exist for all real-valued functions in $\exp (\mathcal{B})$ and will be locally continuous there.

Since $1 \in \exp (\mathcal{B})$ and since $\exp (\mathcal{B})$ is open, there exists an $\varepsilon>0$ such that the neighborhood $U_{\varepsilon}(\mathbf{1}):=\left\{f=e^{g}: g \in \mathcal{B},\|g\|_{\mathcal{B}}<\varepsilon\right\}$ of $\mathbf{1}$ is a subset of $\exp (\mathcal{B})$.

Definition 4.1. Let $\mathcal{B}$ be an $\mathcal{S}$-algebra. The spectral factorization mapping $\mathfrak{S}$ is said to be continuous at $\mathbf{I}$ if there exists an $\varepsilon>0$ such that for every sequence $\left\{f_{n}\right\}_{n \in \mathbb{N}} \subset U_{\varepsilon}(\mathbf{l})$ with $f_{n} \rightarrow \mathbf{I}$ in $\mathcal{B}$ the sequence $\left\{\mathfrak{S} f_{n}\right\}_{n \in \mathbb{N}}$ of spectral factors exists in $\mathcal{B}$ and converges to $\mathfrak{S}_{\mathfrak{l}}=1$, i.e. $\lim _{n \rightarrow \infty}\left\|\mathfrak{S} f_{n}-\mathbf{1}\right\|_{\mathcal{B}}=0$.

THEOREM 4.2. Let $\mathcal{B}$ be an $\mathcal{S}$-algebra on which the spectral factorization mapping $\mathfrak{S}$ is continuous at $\mathbf{1}$. Then the Riesz projection $\mathfrak{P}_{+}$is a bounded mapping $\mathcal{B} \rightarrow \mathcal{B}_{+}$. 
Proof. Since $\mathfrak{S}$ is assumed to be continuous at 1 , there exists an $\varepsilon>0$ such that $\mathfrak{S} f$ exists in $\mathcal{B}$ for each $f=e^{g}$ with $g \in \mathcal{B}$ and $\|g\|_{\mathcal{B}}<\varepsilon$. Let $\left\{g_{n}\right\}_{n \in \mathbb{N}} \subset \mathcal{B}$ be an arbitrary sequence of real-valued functions with $\left\|g_{n}\right\|_{\mathcal{B}}<\varepsilon$ and $\lim _{n \rightarrow \infty}\left\|g_{n}\right\|_{\mathcal{B}}=0$. Then the continuity of the exponential function implies that $\lim _{n \rightarrow \infty}\left\|\exp g_{n}-1\right\|_{\mathcal{B}}=0$, and the continuity of $\mathfrak{S}$ at 1 implies

$$
\lim _{n \rightarrow \infty}\left\|\left[\exp g_{n}\right]_{+}-1\right\|_{\mathcal{B}}=0 .
$$

We choose an arbitrary $g \in \mathcal{B}$ and define $g_{\mu}:=\mu g$ with a positive real number $\mu$ with $\mu<\varepsilon$. Obviously $\left\|g_{\mu}\right\|_{\mathcal{B}} \rightarrow 0$ as $\mu \rightarrow 0$. By (4.1) for every $\delta>0$ there exists a $\mu_{0}>0$ such that

$$
\left\|\left[\exp g_{\mu}\right]_{+}-\mathbf{1}\right\|_{\mathcal{B}}<\delta
$$

for all $\mu \leq \mu_{0}$. Set $f_{\mu}:=\exp g_{\mu}$ and consider

$$
h_{N}:=\sum_{k=1}^{N} \frac{(-1)^{k}}{k}\left[\left(f_{\mu}\right)_{+}-\mathbf{1}\right]^{k} .
$$

Then for all $M>N$ and all $\mu \leq \mu_{0}$,

$$
\left\|h_{M}-h_{N}\right\|_{\mathcal{B}} \leq \sum_{k=N+1}^{M} \frac{1}{k}\left\|\left(f_{\mu}\right)_{+}-\mathbf{1}\right\|_{\mathcal{B}}^{k} \leq \frac{1}{N+1} \sum_{k=N+1}^{M} \delta^{k} \leq \frac{1}{N+1} \frac{1}{1-\delta} .
$$

Thus $\left\{h_{N}\right\}_{N \in \mathbb{N}}$ is a Cauchy sequence in $\mathcal{B}$ which converges to $\log \left[\left(f_{\mu}\right)_{+}\right]$in $\mathcal{B}$. On the other hand, the spectral factor $\left(f_{\mu}\right)_{+}$is given by (2.4). Moreover,

$$
\left[\log \left(f_{\mu}\right)_{+}\right](\zeta)=\frac{\mu}{2} \frac{1}{2 \pi} \int_{-\pi}^{\pi} g\left(e^{i \tau}\right) \frac{e^{i \tau}+\zeta}{e^{i \tau}-\zeta} d \tau=: \frac{\mu}{2}(\mathfrak{R} g)(\zeta) \quad(\zeta \in \mathbb{T})
$$

and since $\log \left[\left(f_{\mu}\right)_{+}\right] \in \mathcal{B}$, this shows that $\mathfrak{R} g \in \mathcal{B}$ for every $g \in \mathcal{B}$. The mapping $\mathfrak{R}: \mathcal{B} \rightarrow \mathcal{B}_{+}$can also be written $[7, \S I I I .1]$ as

$$
(\mathfrak{R} g)(\zeta)=g(\zeta)+i(\mathfrak{H} g)(\zeta) \quad(\zeta \in \mathbb{T})
$$

with the Hilbert transform $\mathfrak{H} g$ of $g$ which is given by

$$
(\mathfrak{H} g)(\zeta)=\sum_{k=-\infty}^{\infty}-i \operatorname{sgn}(k) \widehat{g}(k) \zeta^{k} \quad \text { with } \quad \operatorname{sgn}(k)= \begin{cases}1, & k>0 \\ 0, & k=0 \\ -1, & k<0\end{cases}
$$

or which may be expressed as the principal value integral

$$
(\mathfrak{H} g)\left(e^{i t}\right)=\lim _{\varepsilon \rightarrow 0} \frac{1}{2 \pi} \int_{\varepsilon<|\tau| \leq \pi} \frac{g\left(e^{i[t+\tau]}\right)}{\tan (\tau / 2)} d \tau .
$$

Since $\mathfrak{R} g \in \mathcal{B}$ for every $g \in \mathcal{B},(4.3)$ shows that $\mathfrak{H} g \in \mathcal{B}$ whenever $g \in \mathcal{B}$. Moreover, since $\mathfrak{H} \mathfrak{H} g=-g$ for all $g \in \mathcal{B}_{0}:=\{g \in \mathcal{B}: \widehat{g}(0)=0\}$, it is clear that for every $h \in \mathcal{B}_{0}$ there exists a $g \in \mathcal{B}_{0}$ such that $h=\mathfrak{H} g$. Thus 
$\mathfrak{H}: \mathcal{B}_{0} \rightarrow \mathcal{B}_{0}$ is one-to-one and onto, which implies that the graph of $\mathfrak{H}$ is closed, and that $\mathfrak{H}: \mathcal{B}_{0} \rightarrow \mathcal{B}_{0}$ is continuous by the closed graph theorem. By (4.3), this implies the continuity of $\mathfrak{R}$. The operator $\mathfrak{R}$ can also be expressed in terms of the Riesz projection (e.g. [7, §III.1])

$$
(\mathfrak{R} g)(\zeta)=2\left(\mathfrak{P}_{+} g\right)(\zeta)-\widehat{g}(0) \quad(\zeta \in \mathbb{T})
$$

with the zeroth Fourier coefficient $\widehat{g}(0)$ of $g$. Since $|\widehat{g}(0)| \leq\|g\|_{\infty} \leq\|g\|_{\mathcal{B}}$ and since $\mathfrak{R}$ is bounded on $\mathcal{B}_{0}$, the Riesz projection $\mathfrak{P}_{+}$is bounded on $\mathcal{B}$.

We already saw that if the Riesz projection $\mathfrak{P}_{+}$is bounded on a Banach algebra $\mathcal{A} \subset L^{1}(\mathbb{T})$, then the spectral factorization exists for every $f \in$ $\exp (\mathcal{A})$. Moreover, the assumed continuity of $\mathfrak{S}$ at $\mathbf{l}$ in the previous theorem can be extended to the continuity of $\mathfrak{S}$ at every point $f \in \exp (\mathcal{B})$.

TheOREM 4.3. Let $\mathcal{B}$ be an $\mathcal{S}$-algebra. If the spectral factorization mapping $\mathfrak{S}$ is continuous at $\mathbf{1}$ in $\mathcal{B}$, then $f_{+}=\mathfrak{S} f$ exists for every real-valued $f \in \exp (\mathcal{B})$. Moreover, $\mathfrak{S}$ is locally continuous on $\exp (\mathcal{B})$, i.e. for every real $f \in \exp (\mathcal{B})$ there exist constants $C(f)$ and $r(f)$ such that

$$
\|\mathfrak{S} f-\mathfrak{S} g\|_{\mathcal{B}} \leq C(f)\|f-g\|_{\mathcal{B}}
$$

for all real $g \in \exp (\mathcal{B})$ with $\|f-g\|_{\mathcal{B}}<r(f)$. In particular, one can take

$$
C(f)=\left(2+\|\mathbf{l}\|_{\mathcal{B}}\right)\left\|\mathfrak{P}_{+}\right\|\left\|f^{-1}\right\|_{\mathcal{B}}\|\mathfrak{S} f\|_{\mathcal{B}} \quad \text { and } \quad r(f)=\frac{\|\mathfrak{S} f\|_{\mathcal{B}}}{C(f)} .
$$

Proof. By Theorem 4.2, the Riesz projection is bounded on $\mathcal{B}$. Therefore Proposition 2.6 implies that every real-valued $f \in \exp (\mathcal{B})$ possesses a spectral factorization $f_{+}=\mathfrak{S} f$ in $\mathcal{B}$ which is given by

$$
(\mathfrak{S} f)(z)=\exp \left[\left(\mathfrak{P}_{+} \log f\right)(z)-\frac{1}{2}\left(\mathfrak{P}_{+} \log f\right)(0)\right] \quad(z \in \mathbb{D}) .
$$

Choose $f_{1}, f_{2} \in \exp (\mathcal{B})$ and denote by $q_{1}$ and $q_{2}$ the corresponding arguments of the exponential function in representation (4.5) of the spectral factor. Then $\mathfrak{S} f_{2}-\mathfrak{S} f_{1}=\exp \left(q_{1}\right)\left[\exp \left(q_{2}-q_{1}\right)-\mathbf{1}\right]$ and the continuity of the exponential function implies

$$
\begin{aligned}
\left\|\mathfrak{S} f_{2}-\mathfrak{S} f_{1}\right\|_{\mathcal{B}} & \leq\left\|\exp q_{1}\right\|_{\mathcal{B}}\left\|\exp \left(q_{2}-q_{1}\right)-\mathbf{1}\right\|_{\mathcal{B}} \\
& \leq 2\left\|\mathfrak{S} f_{1}\right\|_{\mathcal{B}}\left\|q_{2}-q_{1}\right\|_{\mathcal{B}}
\end{aligned}
$$

provided that $\left\|q_{2}-q_{1}\right\|_{\mathcal{B}}<1$. Next, we investigate $\left\|q_{2}-q_{1}\right\|_{\mathcal{B}}$. We have

$$
\left\|q_{2}-q_{1}\right\|_{\mathcal{B}} \leq\left\|\mathfrak{P}_{+}\left[\log f_{2}-\log f_{1}\right]\right\|_{\mathcal{B}}-\frac{1}{2}\left|\mathfrak{P}_{+}\left[\log f_{2}-\log f_{1}\right](0)\right|\|\mathbf{l}\|_{\mathcal{B}}
$$

just by the linearity of $\mathfrak{P}_{+}$. To get an upper bound for the second term on the right hand side, we note that $\left|\left(\mathfrak{P}_{+} f\right)(0)\right| \leq\left\|\mathfrak{P}_{+} f\right\|_{\infty} \leq\left\|\mathfrak{P}_{+} f\right\|_{\mathcal{B}}$ for every $f \in \mathcal{B}$ using the maximum modulus principle for analytic functions, and that $\mathcal{B}$ is continuously embedded in $\mathcal{C}(\mathbb{T})$. Together with the boundedness of $\mathfrak{P}_{+}$, one obtains

$$
\left\|q_{2}-q_{1}\right\|_{\mathcal{B}} \leq\left(1+\frac{1}{2}\|\mathbf{1}\|_{\mathcal{B}}\right)\left\|\mathfrak{P}_{+}\right\|\left\|\log f_{2}-\log f_{1}\right\|_{\mathcal{B}}
$$


where $\left\|\mathfrak{P}_{+}\right\|$is the operator norm of $\mathfrak{P}_{+}: \mathcal{B} \rightarrow \mathcal{B}_{+}$. The continuity of the logarithm (Lemma 2.1) shows

$$
\left\|\log f_{2}-\log f_{1}\right\|_{\mathcal{B}} \leq\left\|f_{1}^{-1}\right\|_{\mathcal{B}}\left\|f_{2}-f_{1}\right\|_{\mathcal{B}}
$$

for all $f_{1}, f_{2} \in \exp (\mathcal{B})$ with $\left\|f_{2}-f_{1}\right\|_{\mathcal{B}}<\left\|f_{1}^{-1}\right\|_{\mathcal{B}}^{-1}$. Combining (4.6), (4.7), and (4.8) one obtains the statement of the theorem with the constants (4.4).

The previous proof implies in particular that the spectral factorization mapping is continuous on every $\mathcal{S}$-algebra on which $\mathfrak{P}_{+}: \mathcal{B} \rightarrow \mathcal{B}_{+}$ is bounded:

COROLlARY 4.4. The spectral factorization mapping $\mathfrak{S}$ is continuous on an $\mathcal{S}$-algebra $\mathcal{B}$ if and only if $\mathcal{B}$ is decomposing.

REMARK 4.5. The "if" part of this corollary was already proved in [9].

5. Boundedness of the spectral factorization mapping. This section investigates the boundedness of the spectral factorization mapping $\mathfrak{S}$ on $\mathcal{S}$-algebras. By the theorem of Fejér-Riesz, the spectral factorization exists on $\mathcal{B}$ for all non-negative trigonometric polynomials. Therefore one can analyze the boundedness of $\mathfrak{S}$ on the set of all polynomials. To this end, for every $N \in \mathbb{N}$ define

$$
C(N, \mathcal{B}):=\sup _{f \in \mathcal{P}_{\operatorname{pos}}(N),\|f\|_{\mathcal{B}} \leq 1}\|\mathfrak{S} f\|_{\mathcal{B}}
$$

the boundedness constant of $\mathfrak{S}$ on $\mathcal{P}(N)$. It is clear that $C(N+1, \mathcal{B}) \geq$ $C(N, \mathcal{B})$ for all $N$. Based on these constants, we can say what we mean by the boundedness of the spectral factorization mapping for all polynomials on $\mathcal{B}$ :

Definition 5.1. Let $\mathcal{B}$ be an $\mathcal{S}$-algebra. The spectral factorization mapping $\mathfrak{S}$ is said to be $p$-bounded on $\mathcal{B}$ if

$$
C(\mathcal{B}):=\sup _{N \in \mathbb{N}} C(N, \mathcal{B})<\infty .
$$

REMARK 5.2. We only require the boundedness of $\mathfrak{S}$ for all polynomials. Since $\mathfrak{S}$ is not necessarily continuous on $\mathcal{B}$, the p-boundedness does not imply the boundedness of $\mathfrak{S}$ on the whole space $\mathcal{B}$, in general. But of course, p-boundedness is a necessary requirement for the boundedness of $\mathfrak{S}$ on $\mathcal{B}$.

Obviously, $\lim _{N \rightarrow \infty} C(N, \mathcal{B})=C(\mathcal{B})$ and

$$
\left\|f_{+}\right\|_{\mathcal{B}}=\|\mathfrak{S} f\|_{\mathcal{B}} \leq C(N, \mathcal{B})
$$

for all $f \in \mathcal{P}_{\text {pos }}(N)$ with $\|f\|_{\mathcal{B}} \leq 1$. Let $f \in \mathcal{P}_{\text {pos }}(N)$, set $g:=f /\|f\|_{\mathcal{B}}$, and apply (5.3) to $g$. This shows that

$$
\left\|f_{+}\right\|_{\mathcal{B}}^{2} \leq C(N, \mathcal{B})^{2}\left\|f_{+} \bar{f}_{+}\right\|_{\mathcal{B}}
$$


for all spectral factors $f_{+} \in \mathcal{F}[\mathcal{P}(N)]$. Next, for $N \in \mathbb{N}$ define the constants

$$
D_{+}(N, \mathcal{B}):=\inf _{\substack{f_{+} \in \mathcal{F}[\mathcal{P}(N)] \\\left\|f_{+}\right\|_{\mathcal{B}}=1}}\left\|f_{+} \bar{f}_{+}\right\|_{\mathcal{B}} \quad \text { and } \quad D_{+}(\mathcal{B}):=\lim _{N \rightarrow \infty} D_{+}(N, \mathcal{B})
$$

By this definition, it is clear that the sequence $D_{+}(N, \mathcal{B})$ is decreasing in $N$ and in view of (5.4) one sees that

$$
C(N, \mathcal{B})=\frac{1}{\sqrt{D_{+}(N, \mathcal{B})}} \quad \text { and } \quad C(\mathcal{B})=\frac{1}{\sqrt{D_{+}(\mathcal{B})}} .
$$

Now, the boundedness condition (5.2) can also be stated in terms of $D_{+}(\mathcal{B})$.

Lemma 5.3. The spectral factorization mapping $\mathfrak{S}$ is $p$-bounded on $\mathcal{B}$ if and only if $D_{+}(\mathcal{B})>0$.

The problem in the definition of $D_{+}(N, \mathcal{B})$ is that the infimum is taken over the set $\mathcal{F}[\mathcal{P}(N)]$ of all functions $f_{+} \in \mathcal{P}_{+}(N)$ which are obtained by a spectral factorization from a polynomial $f \in \mathcal{P}_{\text {pos }}(N)$, but this set is unknown in general. However, we will show next that the p-boundedness condition remains unchanged even if one takes the infimum over all analytic polynomials $f \in \mathcal{P}_{+}(N)$ instead of $\mathcal{F}[\mathcal{P}(N)]$. Therefore, we define the constants

$$
D(N, \mathcal{B}):=\inf _{\substack{f \in \mathcal{P}_{+}(N) \\\|f\|_{\mathcal{B}}=1}}\|f \bar{f}\|_{\mathcal{B}} \text { and } \quad D(\mathcal{B}):=\inf _{\substack{f \in \mathcal{B}_{+} \\\|f\|_{\mathcal{B}}=1}}\|f \bar{f}\|_{\mathcal{B}}
$$

It is immediately clear that $D(N, \mathcal{B}) \leq D_{+}(N, \mathcal{B})$ and $D(\mathcal{B}) \leq D_{+}(\mathcal{B})$. Moreover, $D(\mathcal{B})=\lim _{N \rightarrow \infty} D(N, \mathcal{B})$.

Now, we are able to give necessary and sufficient conditions for the pboundedness of the spectral factorization mapping $\mathfrak{S}$ on $\mathcal{S}$-algebras:

Proposition 5.4. Let $\mathcal{B}$ be an $\mathcal{S}$-algebra. Then the spectral factorization mapping $\mathfrak{S}$ is p-bounded on $\mathcal{B}$ if and only if

$$
D(\mathcal{B})=\inf _{g \in \mathcal{B}_{+},\|g\|_{\mathcal{B}}=1}\|g \bar{g}\|_{\mathcal{B}}>0 .
$$

Proof. If (5.6) is satisfied, then $D_{+}(\mathcal{B}) \geq D(\mathcal{B})>0$ and Lemma 5.3 implies the p-boundedness of $\mathfrak{S}$.

The necessity of (5.6) is shown by contradiction. Suppose that $\mathfrak{S}$ is p-bounded but $D(\mathcal{B})=0$. Fix $N \in \mathbb{N}$ and let $D(N, \mathcal{B})$ be defined by (5.5). Then for every $\delta>0$ there exists a $g \in \mathcal{P}_{+}(N)$ with $\|g\|_{\mathcal{B}}=1$ and

$$
\|g \bar{g}\|_{\mathcal{B}} \leq D(N, \mathcal{B})+\delta .
$$

Moreover, since $|g(\zeta) \overline{g(\zeta)}|=|g(\zeta)|^{2}$ for all $\zeta \in \mathbb{T}$, Proposition 3.3 implies that $\|g\|_{\infty}^{2}=\|g \bar{g}\|_{\infty} \leq\|g \bar{g}\|_{\mathcal{B}}$. Together with (5.7) this gives

$$
\|g\|_{\infty} \leq \sqrt{D(N, \mathcal{B})+\delta} .
$$


Let $\mu>0$ and define

$$
g_{\mu}:=\frac{g+\|g\|_{\infty}+\mu}{\|g+\| g\left\|_{\infty}+\mu\right\|_{\mathcal{B}}} .
$$

It is clear that $g_{\mu} \in \mathcal{P}_{+}(N),\left\|g_{\mu}\right\|_{\mathcal{B}}=1$, and $\left|g_{\mu}(z)\right|>0$ for all $z \in \mathbb{D}$. Therefore $g_{\mu} \in \mathcal{F}[\mathcal{P}(N)]$ is a spectral factor. It satisfies

$$
\left\|g_{\mu} \bar{g}_{\mu}\right\|_{\mathcal{B}}=\frac{\left\|g \bar{g}+\left(\|g\|_{\infty}+\mu\right)(g+\bar{g})+\left(\|g\|_{\infty}+\mu\right)^{2}\right\|_{\mathcal{B}}}{\|g+\| g\left\|_{\infty}+\mu\right\|_{\mathcal{B}}^{2}},
$$

and since $D_{+}(N, \mathcal{B}) \leq\left\|g_{\mu} \bar{g}_{\mu}\right\|_{\mathcal{B}}$, one gets

$$
D_{+}(N, \mathcal{B}) \leq \frac{\|g \bar{g}\|_{\mathcal{B}}+\left(\|g\|_{\infty}+\mu\right)\left(1+c_{1}\right)\|g\|_{\mathcal{B}}+\left(\|g\|_{\infty}+\mu\right)^{2}\|\mathbf{I}\|_{\mathcal{B}}}{\left\|g+\left(\|g\|_{\infty}+\mu\right)\right\|_{\mathcal{B}}^{2}}
$$

for every $\mu>0$. Moreover, since

$$
1=\|g\|_{\mathcal{B}}=\|g+\| g\left\|_{\infty}-\right\| g\left\|_{\infty}\right\|_{\mathcal{B}} \leq\|g+\| g\left\|_{\infty}\right\|_{\mathcal{B}}+\|g\|_{\infty}\left\|_{\mathbf{1}}\right\|_{\mathcal{B}}
$$

one obtains, together with (5.8),

$$
\|g+\| g\left\|_{\infty}\right\|_{\mathcal{B}} \geq 1-\|g\|_{\infty}\|\mathbf{1}\|_{\mathcal{B}} \geq 1-\|\mathbf{l}\|_{\mathcal{B}} \sqrt{D(N, \mathcal{B})+\delta} .
$$

Letting $\mu \rightarrow 0$ in (5.9), using $\|g\|_{\mathcal{B}}=1$, and applying the bounds (5.7), (5.8), and (5.10) one deduces from (5.9) that

$$
D_{+}(N, \mathcal{B}) \leq \frac{\left(1+\|\mathbf{1}\|_{\mathcal{B}}\right)[D(N, \mathcal{B})+\delta]+\left(1+c_{1}\right) \sqrt{D(N, \mathcal{B})+\delta}}{1-\|\mathbf{1}\|_{\mathcal{B}} \sqrt{D(N, \mathcal{B})+\delta}}
$$

for all $\delta>0$. Since $D(\mathcal{B})=0$ and $\lim _{N \rightarrow \infty} D(N, \mathcal{B})=D(\mathcal{B})$, the right hand side of (5.11) converges to zero, which shows that

$$
D_{+}(\mathcal{B})=\lim _{N \rightarrow \infty} D_{+}(N, \mathcal{B})=0 .
$$

This contradicts the assumption that $\mathfrak{S}$ is bounded since by Lemma 5.3 the boundedness implies that $D_{+}(\mathcal{B})>0$.

Let $\mathcal{B}$ be an $\mathcal{S}$-algebra on which the spectral factorization mapping is pbounded, let $f \in \mathcal{B}_{+}$, and define $g:=f /\|f\|_{\mathcal{B}}$. Then $g \in \mathcal{B}_{+},\|g\|_{\mathcal{B}}=1$, and since $\mathfrak{S}$ is p-bounded, Proposition 5.4 implies that $\|g \bar{g}\|_{\mathcal{B}}=\|f\|_{\mathcal{B}}^{-2}\|f \bar{f}\|_{\mathcal{B}} \geq$ $D(\mathcal{B})>0$. This shows that

$$
D(\mathcal{B})\|f\|_{\mathcal{B}}^{2} \leq\|f \bar{f}\|_{\mathcal{B}} \quad \text { for all } f \in \mathcal{B}_{+} .
$$

Recall that an inner function is a $\varphi \in\left[L^{\infty}(\mathbb{T})\right]_{+}$such that $|\varphi(\zeta)|=1$ for almost all $\zeta \in \mathbb{T}[7,8]$. Assume that $\varphi \in \mathcal{B}$ is an inner function. Then $\varphi \in \mathcal{B}_{+}$ and $\varphi \bar{\varphi}=1$. Since $\mathfrak{S}$ is assumed to be p-bounded on $\mathcal{B},(5.12)$ implies that there exists a universal upper bound on the norm of every inner function in $\mathcal{B}$,

$$
\|\varphi\|_{\mathcal{B}} \leq \sqrt{\|\mathbf{I}\|_{\mathcal{B}} / D(\mathcal{B})}=: c_{2}
$$


which depends only on the algebra $\mathcal{B}$. For $n=0,1,2, \ldots$ define the functions $s_{n}(z):=z^{n}$. It is clear that $s_{n} \in \mathcal{B}_{+}$for all $n \in \mathbb{N}$, and each $s_{n}$ is an inner function. Moreover, the functions $s_{-n}:=\bar{s}_{n}, n \in \mathbb{N}$, belong to $\mathcal{B}$ and from Proposition 3.4 one obtains a uniform upper bound

$$
\left\|s_{n}\right\|_{\mathcal{B}} \leq c_{1} c_{2} \quad \text { for all } n \in \mathbb{Z} \text {. }
$$

Based on these considerations the following lemma will show that on every $\mathcal{S}$-algebra $\mathcal{B}$ on which $\mathfrak{S}$ is p-bounded, the expression (5.6) is bounded away from zero even if the infimum is taken over all $f \in \mathcal{B}$.

Lemma 5.5. Let $\mathcal{B}$ be an $\mathcal{S}$-algebra on which $\mathfrak{S}$ is p-bounded. Then there exists a constant $c_{3}>0$ such that

$$
c_{3}\|f\|_{\mathcal{B}}^{2} \leq\|f \bar{f}\|_{\mathcal{B}} \quad \text { for all } f \in \mathcal{B}
$$

or equivalently that

$$
\inf _{f \in \mathcal{B},\|f\|_{\mathcal{B}}=1}\|f \bar{f}\|_{\mathcal{B}} \geq c_{3}>0
$$

Proof. Let $f \in \mathcal{B}$. Since the trigonometric polynomials are dense in $\mathcal{B}$, there exists a sequence $\left\{p_{n}\right\}_{n=1}^{\infty}$ of polynomials with $p_{n} \in \mathcal{P}(n)$ such that $\lim _{n \rightarrow \infty}\left\|f-p_{n}\right\|_{\mathcal{B}}=0$. Consequently, for every $\varepsilon>0$ there exists an $N_{0}$ such that

$$
\|f\|_{\mathcal{B}}-\varepsilon \leq\left\|p_{n}\right\|_{\mathcal{B}} \leq\|f\|_{\mathcal{B}}+\varepsilon \quad \text { for all } n \geq N_{0} .
$$

Let $s_{n}(z)=z^{n}$ and define $g_{n}:=s_{n} p_{n}$. Then $g_{n} \in \mathcal{P}_{+}(2 n) \subset \mathcal{B}_{+}$and

$$
\left\|p_{n}\right\|_{\mathcal{B}}=\left\|\bar{s}_{n} s_{n} p_{n}\right\|_{\mathcal{B}} \leq\left\|\bar{s}_{n}\right\|_{\mathcal{B}}\left\|g_{n}\right\|_{\mathcal{B}} \leq c_{1} c_{2}\left\|g_{n}\right\|_{\mathcal{B}}
$$

Since $g_{n} \in \mathcal{B}_{+}$and $\mathfrak{S}$ is p-bounded, Proposition 5.4 implies

$$
D(\mathcal{B})\left\|g_{n}\right\|_{\mathcal{B}}^{2} \leq\left\|g_{n} \bar{g}_{n}\right\|_{\mathcal{B}}=\left\|\bar{s}_{n} g_{n} \cdot s_{n} \bar{g}_{n}\right\|_{\mathcal{B}}=\left\|p_{n} \bar{p}_{n}\right\|_{\mathcal{B}}
$$

Next we consider the expression $f \bar{f}-p_{n} \bar{p}_{n}$. By the triangle inequality together with Proposition 3.4 and (5.15), one obtains

$$
\begin{aligned}
\left\|f \bar{f}-p_{n} \bar{p}_{n}\right\|_{\mathcal{B}} & \leq\|\bar{f}\|_{\mathcal{B}}\left\|f-p_{n}\right\|_{\mathcal{B}}+\left\|p_{n}\right\|_{\mathcal{B}}\left\|\overline{f-p_{n}}\right\|_{\mathcal{B}} \\
& \leq c_{1}\left(\|f\|_{\mathcal{B}}+\left\|p_{n}\right\|_{\mathcal{B}}\right)\left\|f-g_{n}\right\|_{\mathcal{B}} \\
& \leq c_{1}\left(2\|f\|_{\mathcal{B}}+\varepsilon\right)\left\|f-g_{n}\right\|_{\mathcal{B}} .
\end{aligned}
$$

The right hand side of the last inequality converges to 0 as $n \rightarrow \infty$. Therefore, for every $\varepsilon>0$ there exists an $N_{1} \geq N_{0}$ such that

$$
\left\|p_{n} \bar{p}_{n}\right\|_{\mathcal{B}} \leq\|f \bar{f}\|_{\mathcal{B}}+\varepsilon \quad \text { for all } n \geq N_{1} .
$$

Putting together all the previous steps, one obtains, for all $n \geq N_{1}$,

$$
\begin{aligned}
\left(\|f\|_{\mathcal{B}}-\varepsilon\right)^{2} & \stackrel{(5.15)}{\leq}\left\|p_{n}\right\|_{\mathcal{B}}^{2} \stackrel{(5.16)}{\leq}\left(c_{1} c_{2}\right)^{2}\left\|g_{n}\right\|_{\mathcal{B}}^{2} \stackrel{(5.17)}{\leq} \frac{\left(c_{1} c_{2}\right)^{2}}{D(\mathcal{B})}\left\|p_{n} \bar{p}_{n}\right\|_{\mathcal{B}} \\
& \stackrel{(5.18)}{\leq} \frac{\left(c_{1} c_{2}\right)^{2}}{D(\mathcal{B})}\left(\|f \bar{f}\|_{\mathcal{B}}+\varepsilon\right) .
\end{aligned}
$$


Since $\varepsilon$ was arbitrary this shows that

$$
\frac{D(\mathcal{B})}{\left(c_{1} c_{2}\right)^{2}}\|f\|_{\mathcal{B}}^{2} \leq\|f \bar{f}\|_{\mathcal{B}} \quad \text { for all } f \in \mathcal{B}
$$

which is equivalent to the statement of the lemma with $c_{3}=D(\mathcal{B}) /\left(c_{1} c_{2}\right)^{2}$ $>0$.

The following theorem shows that $\mathcal{C}(\mathbb{T})$ is essentially the only $\mathcal{S}$-algebra on which the spectral factorization mapping $\mathfrak{S}$ is bounded.

TheOREM 5.6. Let $\mathcal{B}$ be an $\mathcal{S}$-algebra. If the spectral factorization mapping $\mathfrak{S}$ is p-bounded on $\mathcal{B}$ then $\mathcal{B}$ is (bijectively) isomorphic to $\mathcal{C}(\mathbb{T})$, i.e. there exists a constant $c_{4}$ such that

$$
\|f\|_{\infty} \leq\|f\|_{\mathcal{B}} \leq c_{4}\|f\|_{\infty} \quad \text { for all } f \in \mathcal{B} .
$$

Proof. Proposition 3.3 shows that $\mathcal{B}$ is continuously embedded in $\mathcal{C}(\mathbb{T})$, and it remains to prove the opposite inclusion. To this end, let $h \in \mathcal{B}$ be a real-valued function. Then Lemma 5.5 implies $c_{3}\|h\|_{\mathcal{B}}^{2} \leq\|h \bar{h}\|_{\mathcal{B}}=\left\|h^{2}\right\|_{\mathcal{B}}$. Since $h h \in \mathcal{B}$, Lemma 5.5 can be applied to $h^{2}$, which gives $c_{3}\left\|h^{2}\right\|_{\mathcal{B}}^{2} \leq$ $\left\|h^{4}\right\|_{\mathcal{B}}$. Together with the previous inequality, one gets $c_{3} c_{3}^{2}\|h\|_{\mathcal{B}}^{4} \leq\left\|h^{4}\right\|_{\mathcal{B}}$. Applying this upper bound repeatedly, one obtains

$$
\|h\|_{\mathcal{B}} \leq c_{5}(n)\left\|h^{2^{n}}\right\|_{\mathcal{B}}^{1 / 2^{n}} \quad \text { with } \quad c_{5}(n)=\left(\prod_{k=0}^{n-1} c_{3}^{2^{k}}\right)^{-1 / 2^{n}}=c_{3}^{-\left(2^{n}-1\right) / 2^{n}} .
$$

The spectral radius of $h$ is given by $r_{\sigma}(h)=\lim _{n \rightarrow \infty}\left\|h^{n}\right\|_{\mathcal{B}}^{1 / n}$, and property $(B 4)$ of an $\mathcal{S}$-algebra implies that $\|h\|_{\infty}=r_{\sigma}(h)$. Consequently, for $n \rightarrow \infty$, one obtains

$$
\|h\|_{\mathcal{B}} \leq \frac{1}{c_{3}}\|h\|_{\infty}
$$

for every real-valued $h \in \mathcal{B}$. Now let $f=f_{1}+i f_{2}$ be a complex function in $\mathcal{B}$ with real functions $f_{1}, f_{2} \in \mathcal{B}$. Then it follows from (5.20) that

$$
\|f\|_{\mathcal{B}} \leq \frac{1}{c_{3}}\left(\left\|f_{1}\right\|_{\infty}+\left\|f_{2}\right\|_{\infty}\right) \leq \frac{2}{c_{3}}\|f\|_{\infty}
$$

which is the upper bound in (5.19). Since the trigonometric polynomials are dense in $\mathcal{C}(\mathbb{T})$, for every $\varepsilon>0$ there exists a trigonometric polynomial $p$ such that

$$
\frac{1}{c_{4}}\|f-p\|_{\mathcal{B}} \leq\|f-p\|_{\infty}<\varepsilon
$$

Together with axiom (B3) this shows that $f \in \mathcal{B}$.

It is known that the Riesz projection is unbounded on $\mathcal{C}(\mathbb{T})$ (e.g. $[8$, $\S 9])$. Therefore Theorem 5.6 implies at once that the Riesz projection $\mathfrak{P}_{+}$is unbounded on every $\mathcal{S}$-algebra. 
COROLlary 5.7. Let $\mathcal{B}$ be an $\mathcal{S}$-algebra. If the spectral factorization mapping $\mathfrak{S}$ is p-bounded on $\mathcal{B}$ then the Riesz projection $\mathfrak{P}_{+}$is unbounded on $\mathcal{B}$.

COROLlaRY 5.8. The spectral factorization mapping $\mathfrak{S}$ is not p-bounded on every decomposing $\mathcal{S}$-algebra.

Proof. Let $\mathcal{B}$ be an $\mathcal{S}$-algebra and suppose that $\mathfrak{S}$ is p-bounded on $\mathcal{B}$. Then Corollary 5.7 implies that $\mathfrak{P}_{+}$is unbounded on $\mathcal{B}$ which contradicts the decomposing assumption on $\mathcal{B}$.

REMARK 5.9. Note that "p-bounded" may be replaced by "bounded" in the previous two corollaries, since p-boundedness is a necessary condition for the boundedness of $\mathfrak{S}$ on an $\mathcal{S}$-algebra.

6. Conclusions. In conclusion, the spectral factorization mapping $\mathfrak{S}$ can never be bounded and continuous on an $\mathcal{S}$-algebra $\mathfrak{B}$. Indeed, in order that $\mathfrak{S}$ is bounded on $\mathcal{B}$, it is necessary that the Riesz projection $\mathfrak{P}_{+}$is unbounded on $\mathcal{B}$. But on the other hand, the boundedness of $\mathfrak{P}_{+}$is necessary and sufficient for the continuity of the spectral factorization mapping. This conclusion may be stated as follows.

COROLlary 6.1. Let $\mathcal{B}$ be an $\mathcal{S}$-algebra and let $\mathfrak{S}$ be the spectral factorization mapping on $\mathcal{B}$. Then

- If $\mathfrak{S}$ is continuous on $\mathcal{B}$, then $\mathfrak{S}$ is unbounded.

- If $\mathfrak{S}$ is bounded on $\mathcal{B}$, then $\mathfrak{S}$ is discontinuous.

Thus boundedness and continuity are mutually exclusive for the spectral factorization mapping on every $\mathcal{S}$-algebra.

In applications, one is often interested in the concrete value of the constant (5.1) and its dependence on $N$ since it characterizes the influence of errors in the data on the determined spectral factor. For the Wiener algebra a lower and upper bound for the constant $C(N, \mathcal{W})$ were recently presented in [1]. It would certainly be of interest to know such bounds, if they exist, for general $\mathcal{S}$-algebras.

\section{References}

[1] H. Boche and V. Pohl, Boundedness behavior for the spectral factorization for polynomial data in the Wiener algebra, IEEE Trans. Signal Process. 56 (2008), 31003107 .

[2] - - - On the boundedness of the spectral factorization mapping on decomposing Banach algebras, SIAM J. Control Optim. 47 (2008), 1415-1427.

[3] K. F. Clancey and I. Gohberg, Factorization of Matrix Functions and Singular Integral Operators, Birkhäuser, Basel, 1981.

[4] J. L. Doob, Stochastic Processes, Wiley, New York, 1953. 
[5] L. Fejér, Über nichtnegative trigonometrische Polynome, J. Reine Angew. Math. 146 (1915), 53-82.

[6] B. A. Francis, A Course in $H_{\infty}$ Control Theory, Springer, New York, 1987.

[7] J. B. Garnett, Bounded Analytic Functions, Academic Press, New York, 1981.

[8] K. Hoffman, Banach Spaces of Analytic Functions, Prentice-Hall, Englewood Cliffs, NJ, 1962.

[9] B. Jacob and J. R. Partington, On the boundedness and continuity of the spectral factorization mapping, SIAM J. Control Optim. 40 (2001), 88-106.

[10] T. Kailath, A. H. Sayed, and B. Hassibi, Linear Estimation, Prentice-Hall, Upper Saddle River, NJ, 2000.

[11] S. Korry, Boundedness of Hardy-Littlewood maximal operator in the framework of Lizorkin-Triebel spaces, Rev. Mat. Complut. 15 (2003), 401-416.

[12] H. Luiro, Continuity of the maximal operator in Sobolev spaces, Proc. Amer. Math. Soc. 135 (2006), 243-251.

[13] V. V. Peller and S. V. Khrushchev, Hankel operators, best approximations, and stationary Gaussian processes, Russian Math. Surveys 37 (1982), no. 1, 61-144.

[14] W. Rudin, Functional Analysis, 2nd ed., McGraw-Hill, Boston, 1991.

[15] A. H. Sayed and T. Kailath, A survey of spectral factorization methods, Numer. Linear Algebra Appl. 8 (2001), 467-496.

[16] N. Wiener, Extrapolation, Interpolation, and Smoothing of Stationary Time Series with Engineering Applications, MIT Press and Wiley, 1949.

[17] N. Wiener und E. Hopf, Über eine Klasse singulärer Integralgleichungen, Sitzungsber. Königlich Preuß. Akad. Wiss. Berlin 1931, 696-706.

Heinrich-Hertz Chair

for Mobile Communications

Department of EECS

Technische Universität Berlin

Einsteinufer 25

10587 Berlin, Germany

E-mail: holger.boche@mk.tu-berlin.de
Department of Electrical Engineering Technion - Israel Institute of Technology Haifa 32000, Israel E-mail: pohl@ee.technion.ac.il

Received December 11, 2007

Revised version June 4, 2008 